\title{
Compressive Fracture of Brittle Geomaterial: Fractal Features of Compression-Induced Fracture Surfaces and Failure Mechanism
}

\author{
L. Ren, ${ }^{1,2}$ L. Z. Xie, ${ }^{1,2,3}$ C. B. Li, ${ }^{1,2,3}$ and J. Wang ${ }^{1,2,3}$ \\ ${ }^{1}$ Key Laboratory of Energy Engineering Safety and Mechanics on Disasters (Sichuan University), Ministry of Education, \\ Chengdu 610065, China \\ ${ }^{2}$ School of Architecture and Environment, Sichuan University, Chengdu 610065, China \\ ${ }^{3}$ Institute of New Energy and Low-Carbon Technology, Sichuan University, Chengdu 610065, China
}

Correspondence should be addressed to L. Z. Xie; xielingzhi@scu.edu.cn

Received 9 February 2014; Accepted 3 May 2014; Published 26 May 2014

Academic Editor: Jiangbo Sha

Copyright (C) 2014 L. Ren et al. This is an open access article distributed under the Creative Commons Attribution License, which permits unrestricted use, distribution, and reproduction in any medium, provided the original work is properly cited.

Compressive fracture is one of the most common failure patterns in geotechnical engineering. For better understanding of the local failure mechanism of compressive fractures of brittle geomaterials, three compressive fracture tests were conducted on sandstone. Edge cracked semicircular bend specimens were used and, consequently, fresh and unfilled compressive fracture surfaces were obtained. A laser profilometer was employed to measure the topography of each rough fracture surface, followed by fractal analysis of the irregularities of the obtained compression-induced fracture surfaces using the cubic cover method. To carry out a contrastive analysis with the results of compressive fracture tests, three tension mode fracture tests were also conducted and the fractal features of the obtained fracture surfaces were determined. The obtained average result of the fractal dimensions of the compression-induced surfaces was 2.070, whereas the average result was 2.067 for the tension-induced fracture surfaces. No remarkable differences between the fractal dimensions of the compression-induced and tension-induced fracture surfaces may indicate that compressive fracture may occur, at least on the investigative scale of this work, in a similar manner to tension fracture.

\section{Introduction}

Rock masses are generally discontinuous in nature as a result of various geological processes [1], and usually the presence and properties of discontinuities, such as cracks, flaws, and faults, play a dominant role in the stability and macromechanical behavior of the brittle rock mass. In most geotechnical engineering situations, the rock mass is subjected to compressive loading. Therefore, concerns about the compressive fracture manner of brittle geomaterials are significant and imperative.

So far, many investigations have focused on this meaningful subject from theoretical and experimental viewpoints. For instance, Li and Nordlund [2] developed a constitutive model for brittle rocks based on the analysis of microcracks in order to predict the macroscopic deformation induced by microcracking under compression. In their model, wing cracks were initiated at the tips of sliding cracks and propagated in the major loading direction. According to the process of shear faulting, Gupta and Bergström [3] suggested a model for the compressive failure of rocks, and the presented model addressed the progressive growth of damage that leads to the formation of a critical fault nucleus, which could grow unstably in its own plane by fracturing the grain boundaries in increasingly rapid succession. Horii and Nemat-Nasser [4] investigated the compression-induced microcrack growth in brittle solids. According to their study, it was found that, under axial compression, tension cracks nucleate at the tips of the preexisting model flaw, grow with increasing compression, and become parallel to the direction of the maximum far-field compression. In order to understand the branching process from defects in the geology, a set of branch fractures, formed under uniaxial compressive loading at the tip of a preexisting oblique slot, was studied in PMMA (polymethyl methacrylate) plates by Chaker and Barquins [5]. The mechanism of open hole instability was considered by Germanovich and Dyskin [6] based on the growth of preexisting microfractures in the direction of the greatest 


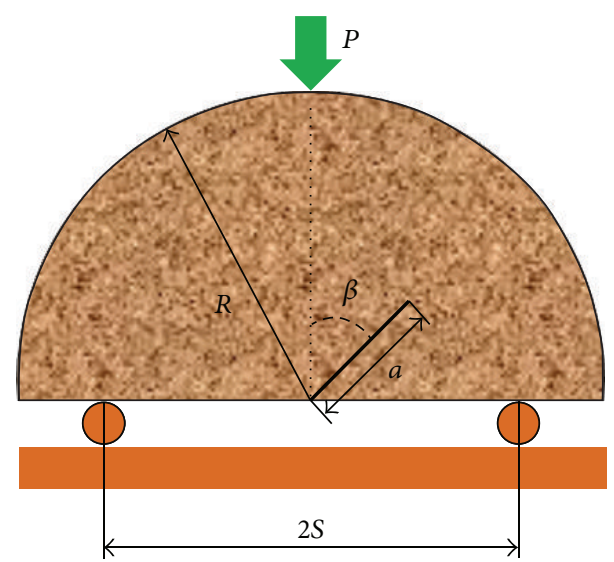

FIGURE 1: Fracture testing configuration of the SCB specimen.

compression. Their results indicated that the major factor enabling the preexisting $3 \mathrm{D}$ cracks to propagate extensively is the presence of the intermediate principal compression near the opening wall.

However, most of the previous studies are focused on the macro- and micromechanisms of crack initiation and propagation under compression, and relatively little is known about the compression-induced fracture surfaces. Particularly for rock-like materials, due to their grainy features, both intergranular and transgranular growths are often involved in macrocrack propagation and such microcrack growth behavior, ultimately, leads to a macro rough fracture surface. Hence, a quantitative description of the topography of a compression-induced fracture surface is very important for a better understanding of the micro failure mechanism of compressive fracture. Fractal geometry [7] was usually employed to characterize a fracture surface, and the rock fracture surfaces indeed exhibit statistical fractal behavior in a certain scale range [8]. The fractal dimension $D$ measures the irregularities and the degree of complexity of the surface shape [9]. However, the data acquisition of a 3D surface is difficult in the early stage [10]. Therefore, a linear profile of a surface and the indirect measurement methods were usually employed to obtain an understanding of the topography features of a 3D surface [11]. By using the noncontact optical instruments, for example, laser profilometer, it became more convenient to obtain the morphology data of a rock fracture surface. Theoretically, the triangular prism surface area method (TPSAM) [12], projective covering method (PCM) [11], and cubic covering method (CCM) [13] enable one to conduct a direct theoretical analysis of a rough fracture surface. Therefore, a real 3D fractal analysis of a fracture surface is being popular in the recent two decades.

The objective of the study was to present a detailed investigation on the micro fracture characters of the compressioninduced fracture surfaces based on fractal analysis. The purpose of the fractal calculations was to indicate the failure mechanism of compressive fracture. Therefore, several compressive and tension mode fracture tests were conducted on sandstone by using edge cracked semicircle bend (SCB) specimens. A laser profilometer was employed to measure the

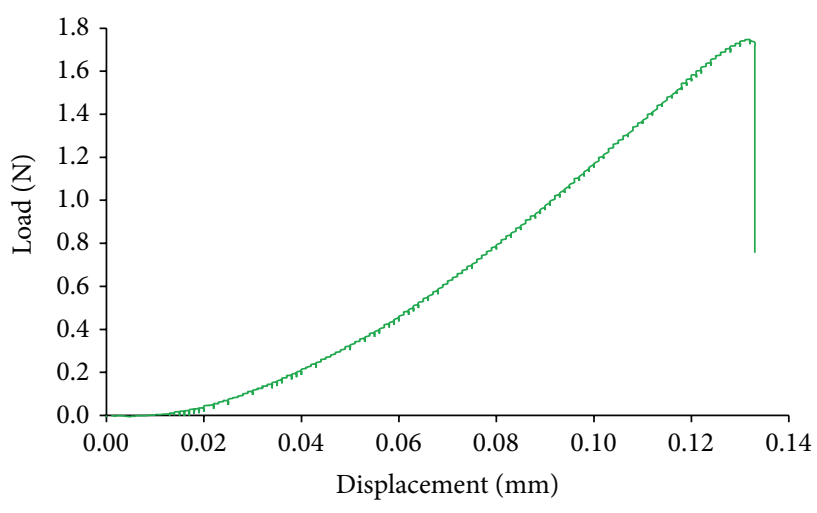

FIGURE 2: Typical load-displacement curve.

topography of each rough fracture surface, followed by fractal analysis of the irregularities of the obtained compressive fracture surfaces, implemented by employing the CCM.

\section{Compression and Tension Mode Fracture Tests on Sandstone}

2.1. Sample Preparation. The SCB specimen $[14,15]$ used in the experiments consists of a half disc of radius $R$ and thickness $B$ with an inclined edge crack of length $a$, where the inclined crack angle is $\beta$. The SCB specimen was loaded under three-point bend with two bottom supports, at a half distance $S$, as shown in Figure 1. In this investigation, both the ratios of $a / R$ and $D / R$ were set to 0.4 . The geometrical factors for this SCB specimen configuration have been reported by Ayatollahi and Aliha [16], and it can be seen that an inclined angle $\beta=40^{\circ}$ corresponds to a compression mode crack for this size of specimen. As a consequence, a crack inclined angle, $\beta=40^{\circ}$, was selected.

The test material, sandstone, was taken from a stone quarry in Fangshan County, Beijing, and had a dry density of about $2.41 \mathrm{~g} / \mathrm{cm}^{3}$. Visual inspections indicated that the sandstone grains were about $0.1 \mathrm{~mm} \sim 0.8 \mathrm{~mm}$ in size. A sandstone core was drilled using a diamond drill bit with a diameter $100 \mathrm{~mm}$. By using a rotary diamond saw, circular disks were then cut from the core and these disks were split into two halves, each of which had a thickness of approximately $15 \mathrm{~mm}$. The half discs were machined until the radius was about $25 \mathrm{~mm}$. Finally, a thin fret saw blade of $0.2 \mathrm{~mm}$ thickness was used for creating the edge cracks in the $\mathrm{SCB}$ specimens along the required inclination angles of $0^{\circ}$ and $40^{\circ}$. For each crack inclination angle, three specimens were prepared. The samples in the tension mode were numbered as $\mathrm{T}-1, \mathrm{~T}-2$, and $\mathrm{T}-3$, and the samples in the compression mode were numbered as $\mathrm{C}-1, \mathrm{C}-2$, and $\mathrm{C}-3$.

2.2. Experiments. The SCB specimens were loaded by a servohydraulic compressive test machine of capacity $10 \mathrm{kN}$. The tests were carried out under displacement control, at a fixed loading rate of $0.15 \mathrm{~mm} / \mathrm{min}$ for all the SCB specimens. A typical plot of the applied load against the load-point displacement is shown in Figure 2. It can be seen that, after 

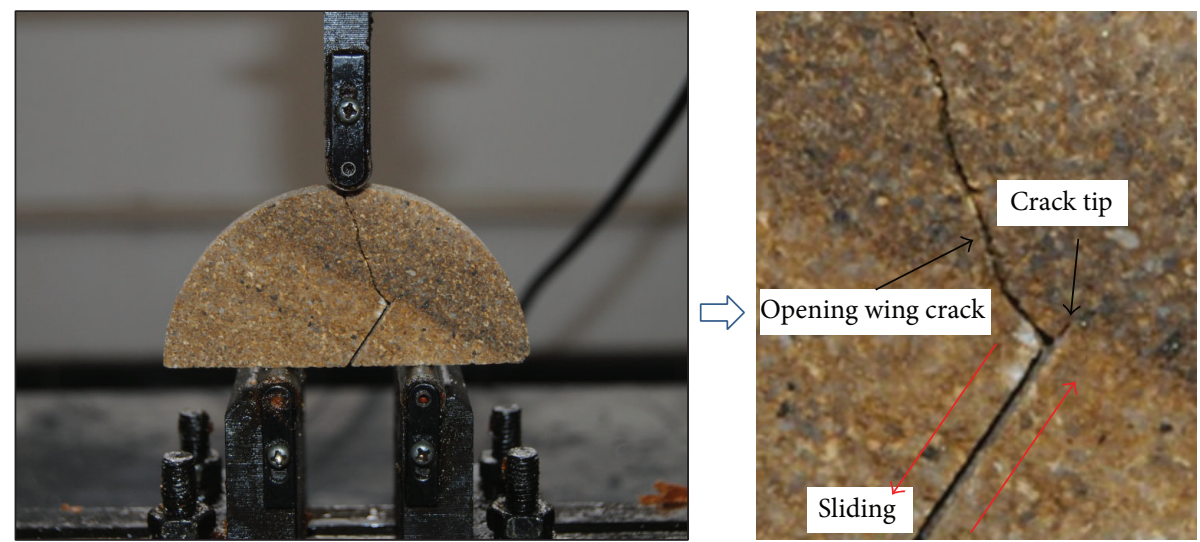

FIGURE 3: Typical broken SCB specimen and the failure pattern of compression-induced fracture.

a nonlinear stage, the load-displacement curve had a linear relationship up to the maximum load, and then followed by a rapidly dropping. Such a sudden failure phenomenon clearly indicates the brittle behavior of sandstone.

When $\beta=0^{\circ}$, the crack is a pure mode I (tension mode) crack, and it propagates straight along the original crack line from the crack tip. For $\beta=40^{\circ}$, the crack is a compression mode crack, and it grows along a curvilinear path. However, the propagation is not initiated from the preexisting crack tip. When the propagating crack reaches the upper boundary of the SCB specimen, the sample breaks into two pieces. A typical totally broken sample in the compression mode is shown in Figure 3.

\section{Fractal Features of Fracture Surfaces for Different Loading Conditions}

3.1. Surface Topography Measurements. Topographical measurement of the fracture surfaces was carried out using a 3D laser profilometer, a noncontact device that enables one to obtain data files of $x, y$, and $z$ coordinates. Its laser probe provides an accuracy of $\pm 0.1 \mathrm{~mm}$ and it has an elevation range of $300 \mathrm{~mm}$. It can move automatically over a sample using a preprogrammed path to measure the topography of the fracture surface. The data set from a fracture surface topography consists of both coordinates and the corresponding heights of the object surface. Each fracture surface was scanned using a $0.1 \mathrm{~mm}$ sampling interval, and all the samples were scanned from the positions where crack initiations happened. Height information at each point was then transferred to the computer and an isometric view of the internal scanning field $(10 \mathrm{~mm} \times 10 \mathrm{~mm})$ was reconstructed. Fracture profile views of each fracture surface are illustrated in Figure 4.

3.2. Covering Method. The CCM [13] provides a simple way to conduct a direct estimation of the fractal dimension of a rough surface. As shown in Figure 5, assuming that there is a regular square grid on the plane $X O Y$ and each grid cell has a scale $\delta$, four intersection points correspond to the four heights of the fracture surface: $h_{1}(i ; j), h_{2}(i ; j+1), h_{3}(i+1 ; j)$, and $h_{4}(i+1 ; j+1)$ (where $1 \leq i, j \leq n-1$, and $n$ is the total number of sampling points on each individual part of the fracture surface). When using cubes with scale $\delta$ to cover the fracture surface, the maximum difference among $h_{1}(i ; j)$, $h_{2}(i ; j+1), h_{3}(i+1 ; j)$, and $h_{4}(i+1 ; j+1)$ will determine the number of cubes needed to cover the irregular surface area within the scale $\delta$. The number $N_{i ; j}$ of cubes needed to cover the fracture surface in the field of the $(i ; j)$ th grid unit on the reference plane $\mathrm{XOY}$ is given by

$$
\begin{aligned}
N_{i, j}=\operatorname{INT}\left\{\delta^{-1}\right. & {\left[\max \left(h_{1}, h_{2}, h_{3}, h_{4}\right)\right.} \\
& \left.\left.-\min \left(h_{1}, h_{2}, h_{3}, h_{4}\right)\right]+1\right\},
\end{aligned}
$$

where INT denotes the integrating function. Then the total number of cubes needed to cover the whole fracture surface is

$$
N(\delta)=\sum_{i, j=1}^{n-1} N_{i, j}
$$

Changing the measurement scale $\delta$, one can get different values of $N(\delta)$. The total number $N(\delta)$ of cubes depends on the measurement interval $\delta$ used.

If the fracture surface appears to be a fractal, the relation between $N(\delta)$ and $\delta$ is

$$
N(\delta) \sim \delta^{-D}
$$

where $D$ is the fractal dimension of the fracture surface.

3.3. Fractal Features of the Fracture Surfaces. The fractal dimensions of the fracture surfaces were calculated using the CCM, and covering scales of $\delta=(0.1 \mathrm{~mm}, 0.2 \mathrm{~mm}, 0.4 \mathrm{~mm}$, $0.5 \mathrm{~mm}, 1.0 \mathrm{~mm}, 2.0 \mathrm{~mm}, 2.5 \mathrm{~mm}, 5.0 \mathrm{~mm}, 10.0 \mathrm{~mm}$ ) were selected. The relation between the total number $N(\delta)$ of cubes needed to cover the whole fracture surface and the sizes $\delta$ of the cubes is given in Table 1. For cases in which $\delta>2.0 \mathrm{~mm}$, all the rough surfaces needed the same number of cubes for each covering scale.

Based on Table 1, the relation between the total number $N(\delta)$ and grid cell size $\delta$ can be plotted on a log-log scale, 

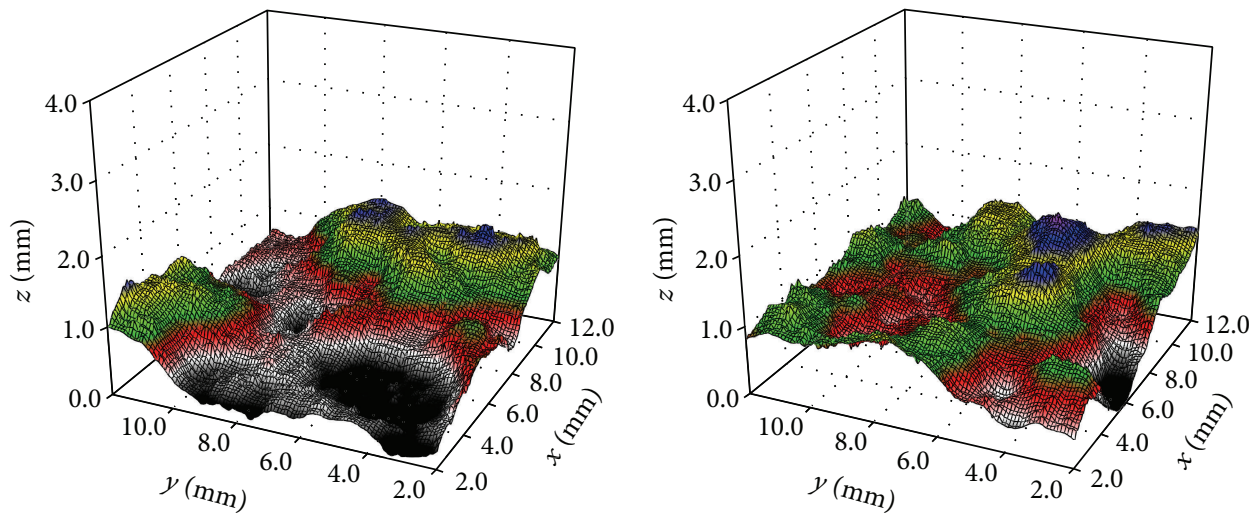

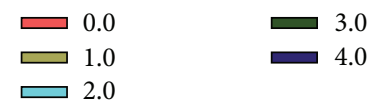

Sample number: T-1

(a)
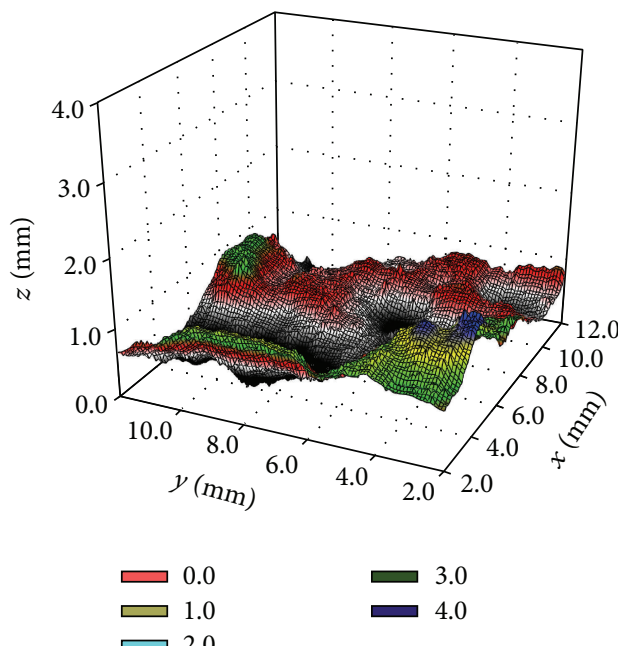

Sample number: T-3

(c)

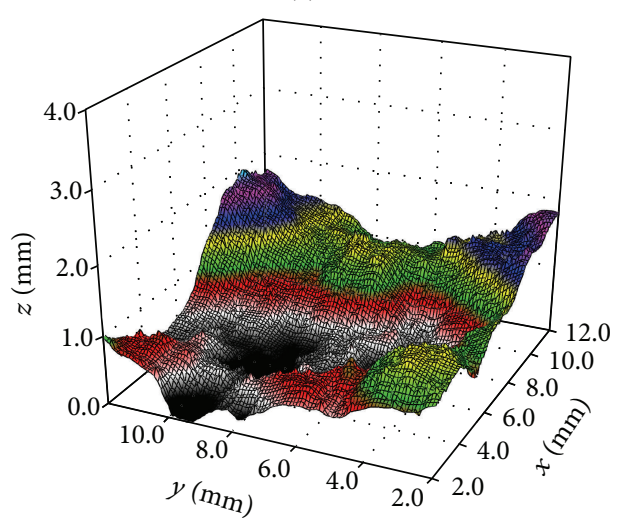

0.0
0
1.0 $\quad \begin{array}{r}3.0 \\ 4.0\end{array}$

ऍ 2.0

Sample number: C-2

(e)

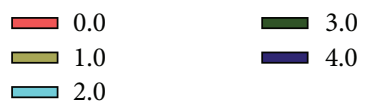

Sample number: T-2
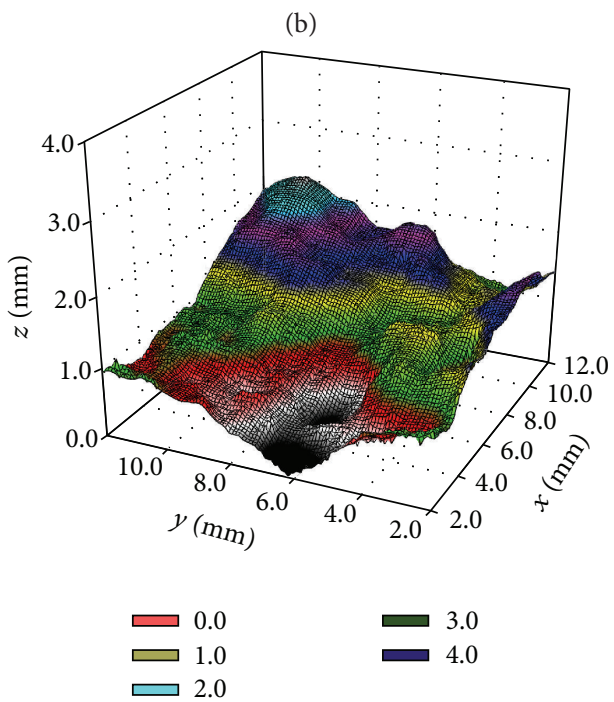

Sample number: C-1

(d)

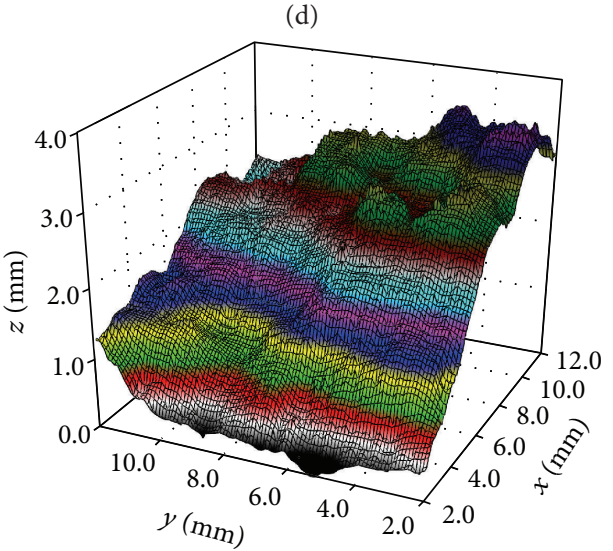

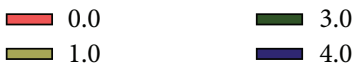

Sample number: C-3

(f)

FIGURE 4: Surface topography plots of the fracture surfaces. 


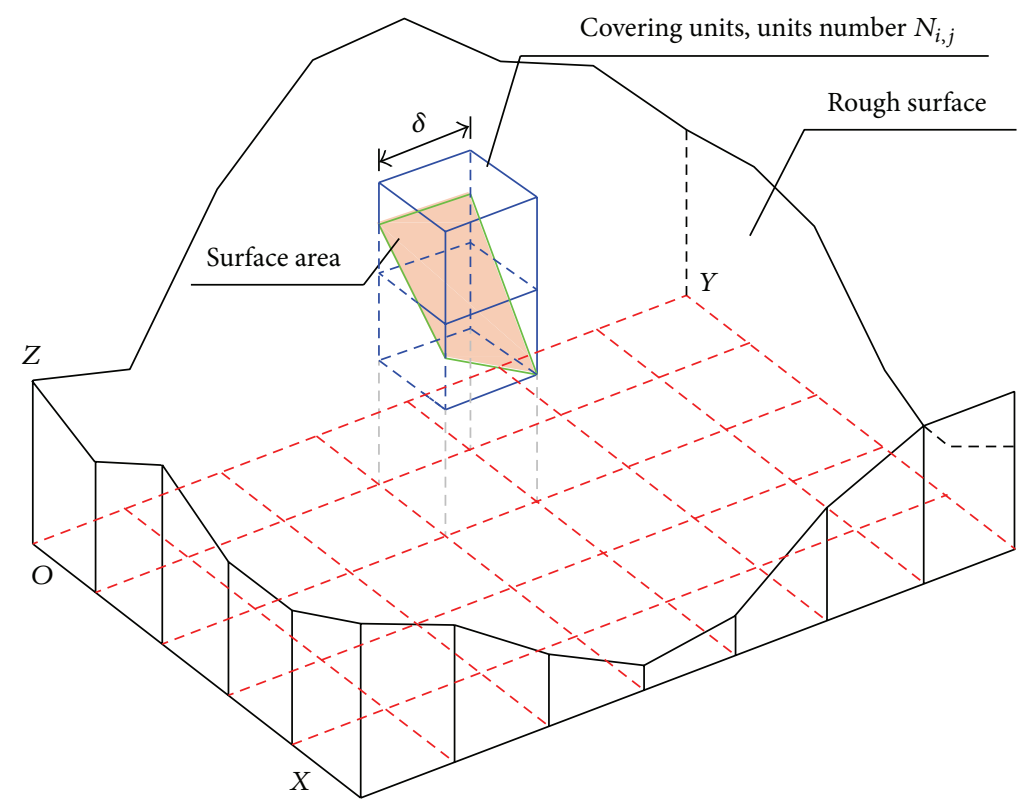

FIGURE 5: Schematic view of the cubic covering method (a redraw version according to [13]).

TABLE 1: Total numbers $N(\delta)$ of required cubes and the measurement scales $\delta$, according to the CCM.

\begin{tabular}{|c|c|c|c|c|c|c|}
\hline \multirow{2}{*}{$\delta / \mathrm{mm}$} & \multicolumn{3}{|c|}{ Tension-induced fracture surfaces } & \multicolumn{3}{|c|}{ Compression-induced fracture surfaces } \\
\hline & No. T-1 & No. T-2 & No. T-3 & No. C-1 & No. C-2 & No. C-3 \\
\hline 0.1 & 11309 & 11197 & 11064 & 10570 & 11294 & 13465 \\
\hline 0.2 & 2591 & 2575 & 2572 & 2567 & 2579 & 2872 \\
\hline 0.4 & 628 & 630 & 629 & 634 & 625 & 669 \\
\hline 0.5 & 401 & 401 & 401 & 404 & 401 & 430 \\
\hline 1.0 & 100 & 100 & 100 & 100 & 100 & 104 \\
\hline 2.0 & 25 & 25 & 25 & 25 & 25 & 25 \\
\hline 2.5 & 16 & 16 & 16 & 16 & 16 & 16 \\
\hline 5.0 & 4 & 4 & 4 & 4 & 4 & 4 \\
\hline 10.0 & 1 & 1 & 1 & 1 & 1 & 1 \\
\hline
\end{tabular}

as shown in Figure 6. If the fracture surfaces exhibit fractal behavior, the values obtained should be between 2.0 and 3.0.

For the surface of sample number T-1, the correlation coefficients of linear regression analysis are 0.9999 on the scale ranging from 0.1 to $0.5 \mathrm{~mm}$ and 1.0 on the scale ranging from 1.0 to $10.0 \mathrm{~mm}$, respectively. On the scale of 0.1 to $0.5 \mathrm{~mm}$, the fractal dimension of the fracture surface is 2.074 , while on the scale of 1.0 to $10.0 \mathrm{~mm}$, the fractal dimension is 2.0. Actually, for each fracture surface, it was found that the data points in the log-log plot do not fall onto a straight line; thus, there is no universal fractal dimension on all scales for a fracture surface. The same conclusion was drawn by Zhou and Xie [13]. Additionally, it should be noted that the selected scale range has a great influence on the calculated result of the fractal dimension. For instance, for sample T-1, on a scale ranging from 0.1 to $0.2 \mathrm{~mm}$, the calculated fractal dimension is 2.1259; on a scale ranging from 0.1 to $0.4 \mathrm{~mm}$, the calculated result is 2.0853. Similar conclusions can be found for all the tension-induced and compression-induced fracture surfaces. Therefore, only if the covering scale $\delta$ tends to zero, the calculated fractal dimension will be close to the real fractal dimension of a fracture surface.

\section{Discussions on the Failure Mechanism of Compression-Induced Fracture}

4.1. Fracture Mechanism of Sandstone Based on Fractal Analysis. According to the analysis in [17], both wing cracks and secondary cracks were observed in rocks and rock-model materials under compression. Wing cracks usually propagate from the tip of a preexisting crack along a curvilinear path and towards the direction of the maximum compressive stress. From the descriptions in [17], the wing cracks are usually assumed to be tensile cracks. However, a validation of the assumption is missing. Especially for the theoretical modes which depend on such a hypothesis, the corresponded validation is significantly necessary.

From Figure 6, a noticeable difference among the fractal dimensions for the compression-induced surfaces was observed. Noting that the grain size of this sandstone was 


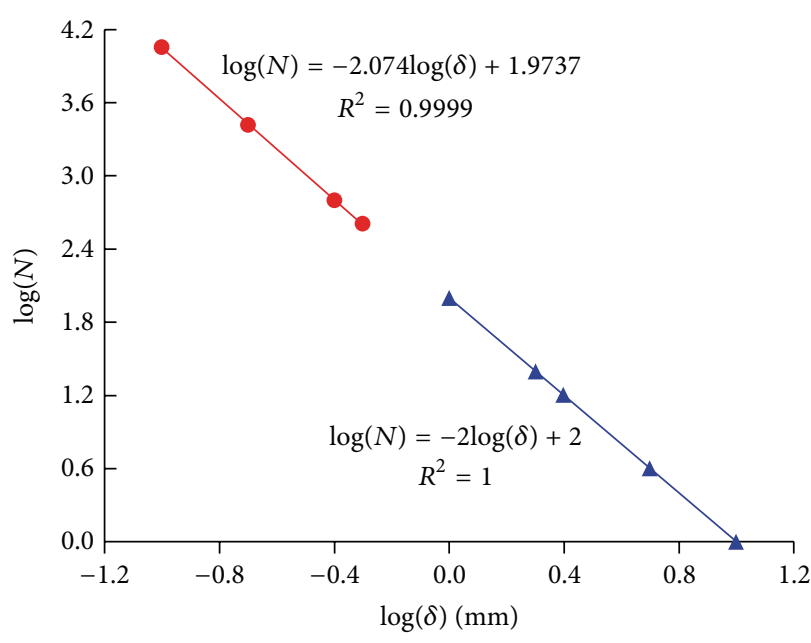

Sample number: T-1

(a)

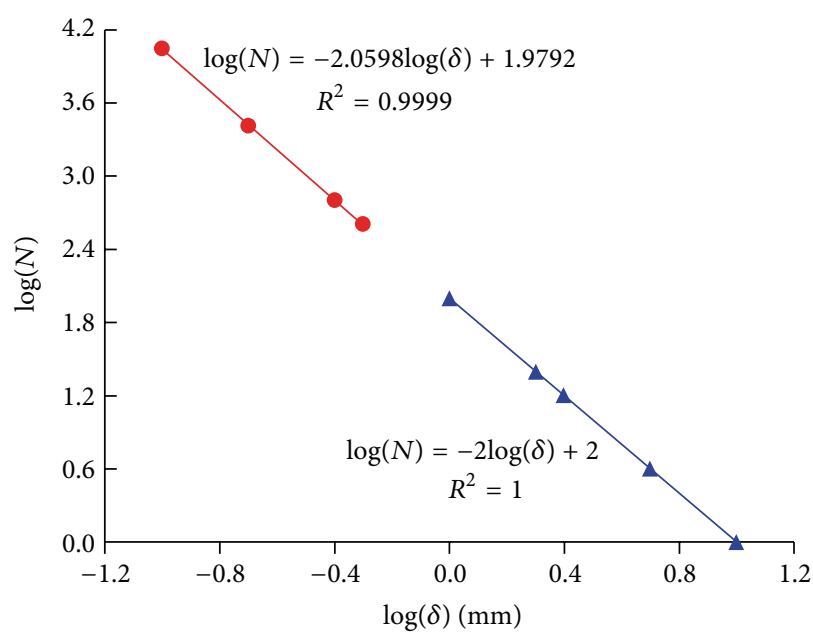

Sample number: T-3

(c)

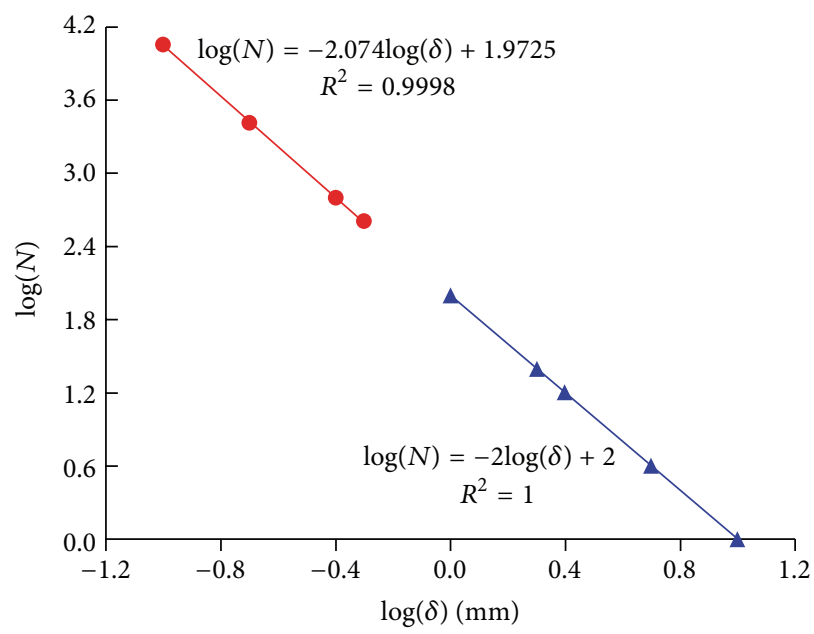

Sample number: C-2

(e)

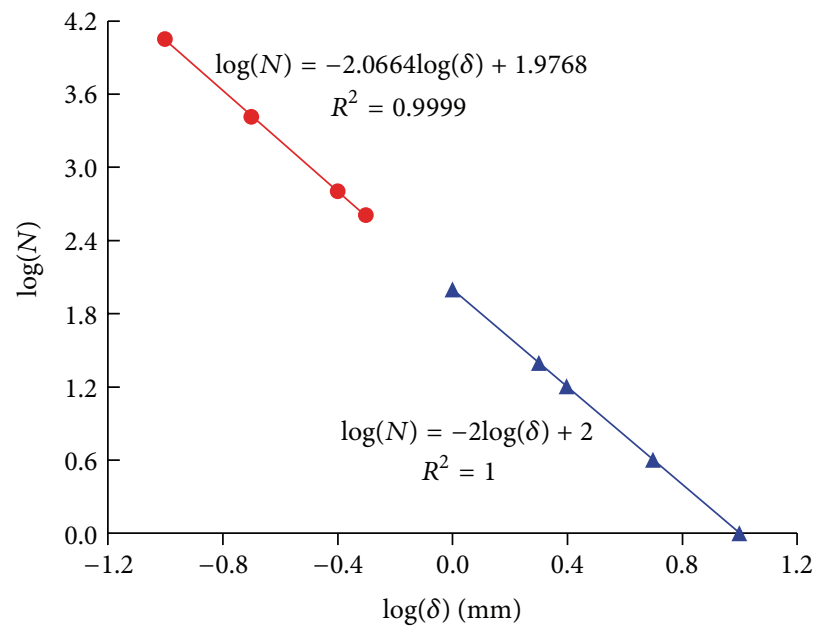

Sample number: T-2

(b)

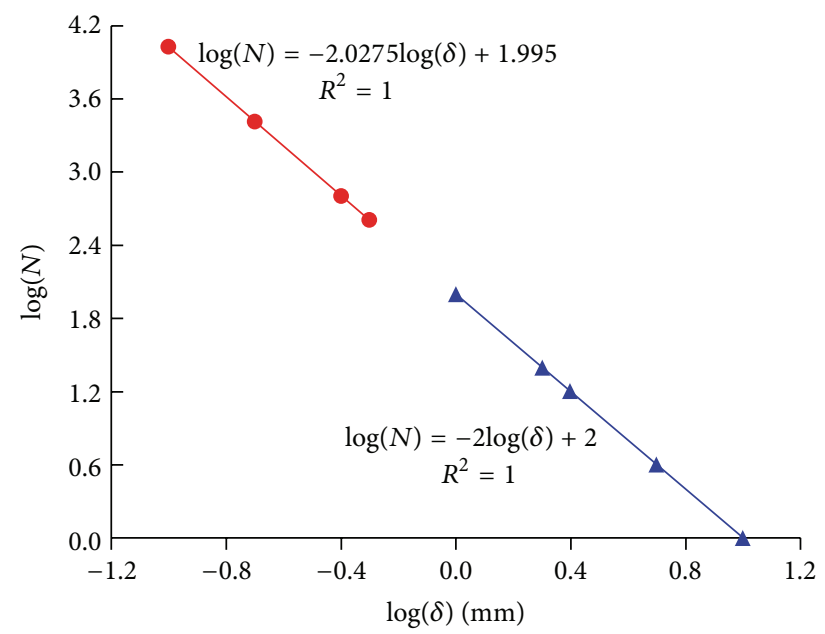

Sample number: C-1

(d)

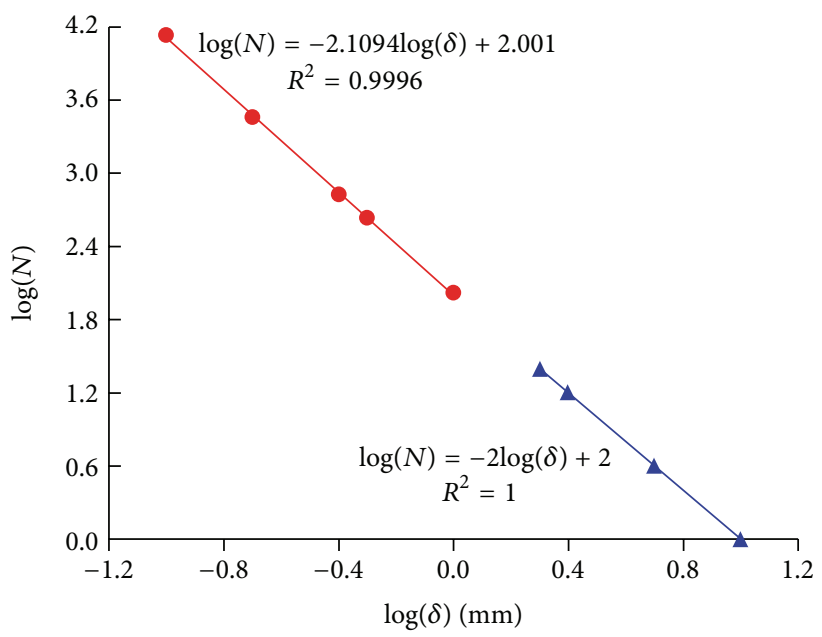

Sample number: C-3

(f)

Figure 6: Log-log plots of $N(\delta)$ and $\delta$ for each fracture surface. 
between 0.1 and $0.8 \mathrm{~mm}$, as discussed in [18], the inconsistencies are related to the petrological-mineralogical and petrophysical properties of the rock samples. However, the average result of the fractal dimensions of the compressioninduced surfaces was 2.070, whereas the average result was 2.067 for the tension-induced fracture surfaces. Therefore, regardless of the measurement error and the mathematical error, no remarkable differences were found between the fractal dimensions of the compression-induced and tensioninduced fracture surfaces. As is known, the fractal dimension describes the irregularity of the surface roughness, and a higher fractal dimension corresponds to a rougher fracture surface [18]. Therefore, the results in the current study show that, at least on a scale of millimeters, the roughness of the fracture surfaces seems to be an invariant. For the tension mode fracture, the rock mass ahead of the crack was highly stretched. Due to almost the same fractal dimensions of the tension-induced and compression-induced fracture surfaces, it seems that the compression-induced fracture in the SCB specimen was also caused by stretching. Therefore, the assumption that wing cracks were induced by tensile stress is, to some extent, reasonable.

4.2. On the Initiation of Compressive Fracture. The critical condition and initiation angle are important for investigating the strength of fractured rock masses. For a SCB specimen with an inclined angle $\beta=40^{\circ} \mathrm{crack}$, the compressive fracture occurs, essentially, under compression-shear loading on the original crack plane. And in such case, from a view of fracture mechanics, wing crack will initiate from the crack tip along the direction of $\theta_{c}>\theta_{0}$, where $\theta_{0}$ is the initiation angle of pure mode II crack. However, in this study, no wing cracks were observed to initiate from the preexisting cracks, as shown in Figure 3. Actually, the results in [5] also confirmed that wing cracks may not necessarily initiate from preexisting cracks, but from a certain distance from the initial crack tip. It must be clarified that the cracks introduced in this work are not perfect cracks although their thickness is almost only $0.2 \mathrm{~mm}$, and this may be a reason that wing cracks did not initiate from the original crack tips. Additionally, as the inclined crack angle $\beta$ tends to $90^{\circ}$, the crack, or strictly speaking the notch, will close and the sliding deformation of the notch front becomes negligible. In such case, it is impossible that the crack could start from the notch front, but it started at the center of the bottom of the specimen and it propagates along the loading direction. Similar experimental results can be also found in [19].

Nevertheless, whether the wing crack initiates at the preexisting crack tip or not, the wing cracks are usually subjected to tensile loading. Hence, the fractal dimensions of tension-induced fracture surfaces should coincide with the results of compression-induced fracture surfaces and this has been confirmed in our work.

\section{Conclusions}

Aiming at the missing of the knowledge of compressioninduced fracture surfaces, we have applied the cubic cover method to analyze compression-induced fracture surfaces in this study in detail. Additionally, to validate the assumption, that is, the wing cracks are usually assumed to be tensile cracks, adopted in some theoretical models, we also present an analysis of the local failure mechanism in the compressive fracture of sandstone. It was found that

(i) the data points in the log-log plot of $N$ versus $\delta$ do not fall onto a straight line for both the tension-induced and compression-induced fracture surfaces;

(ii) the selected scale range has a great influence on the calculated result of the fractal dimensions;

(iii) the petrological-mineralogical and petrophysical properties of the rock samples may result in a noticeable difference between the fractal dimensions for compression-induced surfaces;

(iv) almost the same fractal dimensions for the tensioninduced and compression-induced fracture surfaces were obtained;

(v) both the tension-induced and the compressioninduced fracture surfaces were essentially caused by tensile stresses in the SCB specimens.

\section{Conflict of Interests}

The authors declare that there is no conflict of interests regarding the publication of this paper.

\section{Acknowledgments}

This work was financially supported by the Provincial Science and Technology Support Project (2012FZ0124), Major State Basic Research Project (2011CB201201), and the International Science \& Technology Cooperation Program of China (2012DFA60760).

\section{References}

[1] O. Mughieda and A. K. Alzo'ubi, "Fracture mechanisms of offset rock joints-a laboratory investigation," Geotechnical \& Geological Engineering, vol. 22, no. 4, pp. 545-562, 2004.

[2] C. Li and E. Nordlund, "Deformation of brittle rocks under compression-with particular reference to microcracks," Mechanics of Materials, vol. 15, no. 3, pp. 223-239, 1993.

[3] V. Gupta and J. S. Bergström, "Compressive failure of rocks," International Journal of Rock Mechanics and Mining Sciences, vol. 34, no. 3-4, pp. 112.e1-112.e23, 1997.

[4] H. Horii and S. Nemat-Nasser, "Compression-induced microcrack growth in brittle solids: axial splitting and shear failure," Journal of Geophysical Research: Solid Earth, vol. 90, pp. 31053125, 1985.

[5] C. Chaker and M. Barquins, "Sliding effect on branch crack," Physics and Chemistry of the Earth, vol. 21, no. 4, pp. 319-323, 1996.

[6] L. N. Germanovich and A. V. Dyskin, "Fracture mechanisms and instability of openings in compression," International Journal of Rock Mechanics and Mining Sciences, vol. 37, no. 1-2, pp. 263-284, 2000. 
[7] B. B. Mandelbrot, The Fractal Geometry of Nature, Henry Holt, New York, NY, USA, 1983.

[8] H. Xie, H. Sun, Y. Ju, and Z. Feng, "Study on generation of rock fracture surfaces by using fractal interpolation," International Journal of Solids and Structures, vol. 38, no. 32-33, pp. 5765-5787, 2001.

[9] W. Kwaśny, "Structure, physical properties and fractal character of surface topography of CVD coatings," Journal of Machine Engineering, vol. 11, no. 1-2, pp. 134-151, 2011.

[10] V. Rasouli and J. P. Harrison, "Assessment of rock fracture surface roughness using Riemannian statistics of linear profiles," International Journal of Rock Mechanics and Mining Sciences, vol. 47, no. 6, pp. 940-948, 2010.

[11] H. Xie, J.-A. Wang, and E. Stein, "Direct fractal measurement and multifractal properties of fracture surfaces," Physics Letters A: General, Atomic and Solid State Physics, vol. 242, no. 1-2, pp. 41-50, 1998.

[12] K. C. Clarke, "Computation of the fractal dimension of topographic surfaces using the triangular prism surface area method," Computers \& Geosciences, vol. 12, no. 5, pp. 713-722, 1986.

[13] H. W. Zhou and H. Xie, "Direct estimation of the fractal dimensions of a fracture surface of rock," Surface Review and Letters, vol. 10, no. 5, pp. 751-762, 2003.

[14] K. P. Chong and M. D. Kuruppu, "New specimen for fracture toughness determination for rock and other materials," International Journal of Fracture, vol. 26, no. 2, pp. R59-R62, 1984.

[15] M. D. Kuruppu and K. P. Chong, "Fracture toughness testing of brittle materials using semi-circular bend (SCB) specimen," Engineering Fracture Mechanics, vol. 91, pp. 133-150, 2012.

[16] M. R. Ayatollahi and M. R. M. Aliha, "Wide range data for crack tip parameters in two disc-type specimens under mixed mode loading," Computational Materials Science, vol. 38, no. 4, pp. 660-670, 2007.

[17] A. Bobet, "The initiation of secondary cracks in compression," Engineering Fracture Mechanics, vol. 66, no. 2, pp. 187-219, 2000.

[18] T. Babadagli and K. Develi, "Fractal characteristics of rocks fractured under tension," Theoretical and Applied Fracture Mechanics, vol. 39, no. 1, pp. 73-88, 2003.

[19] N. A. Al-Shayea, "Crack propagation trajectories for rocks under mixed mode I-II fracture," Engineering Geology, vol. 81, no. 1, pp. 84-97, 2005. 

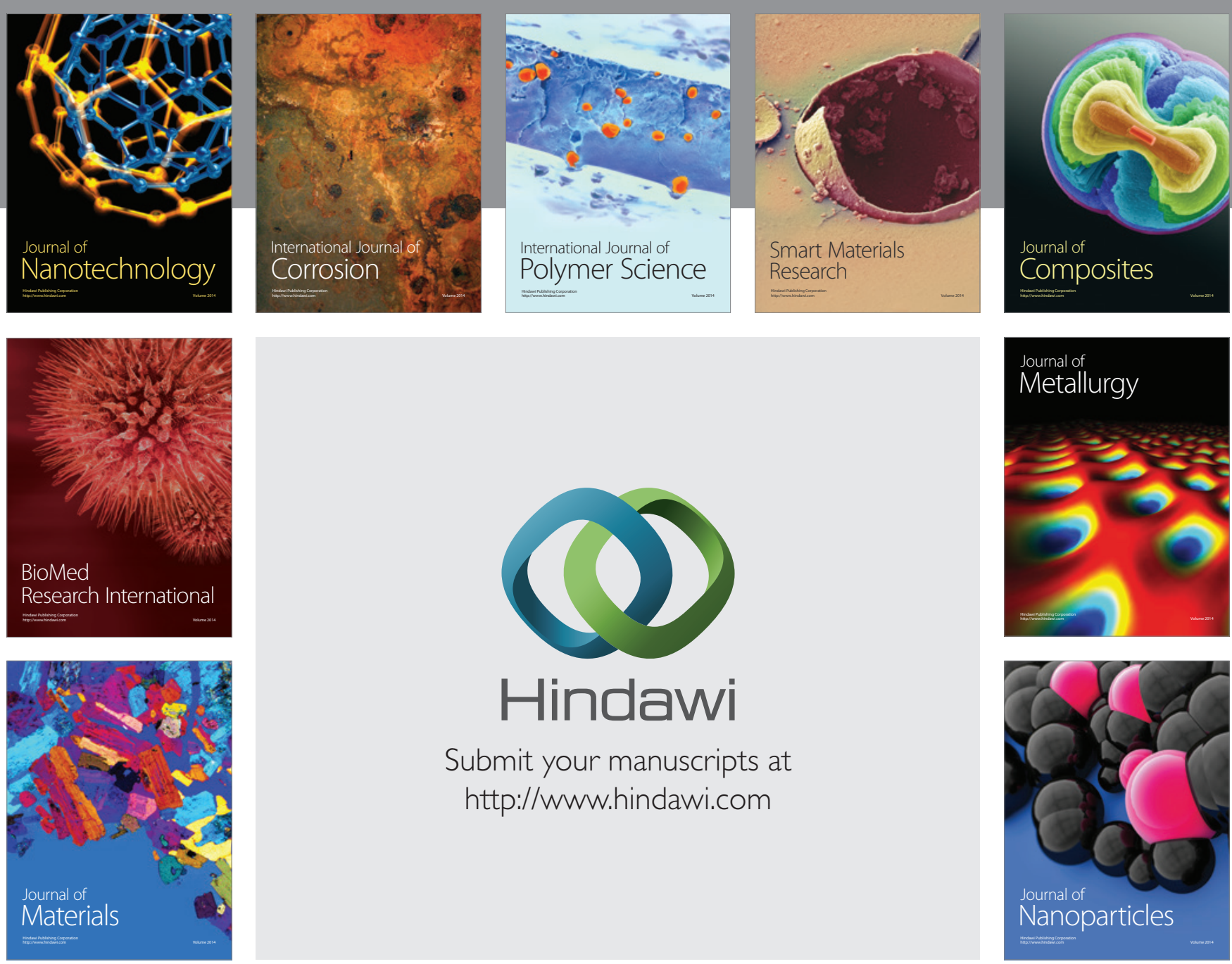

Submit your manuscripts at http://www.hindawi.com
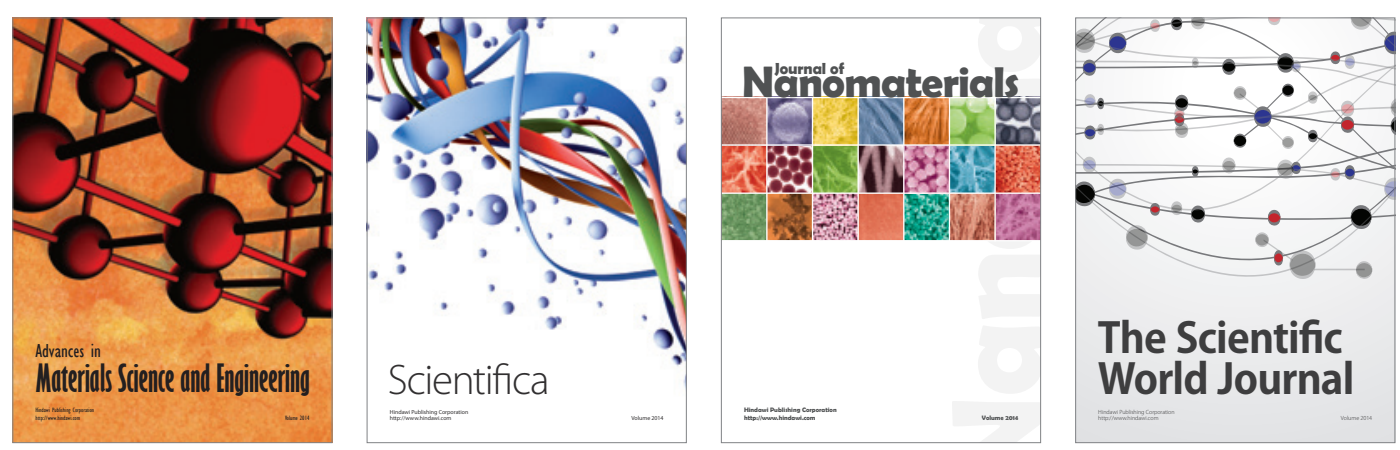

\section{The Scientific World Journal}
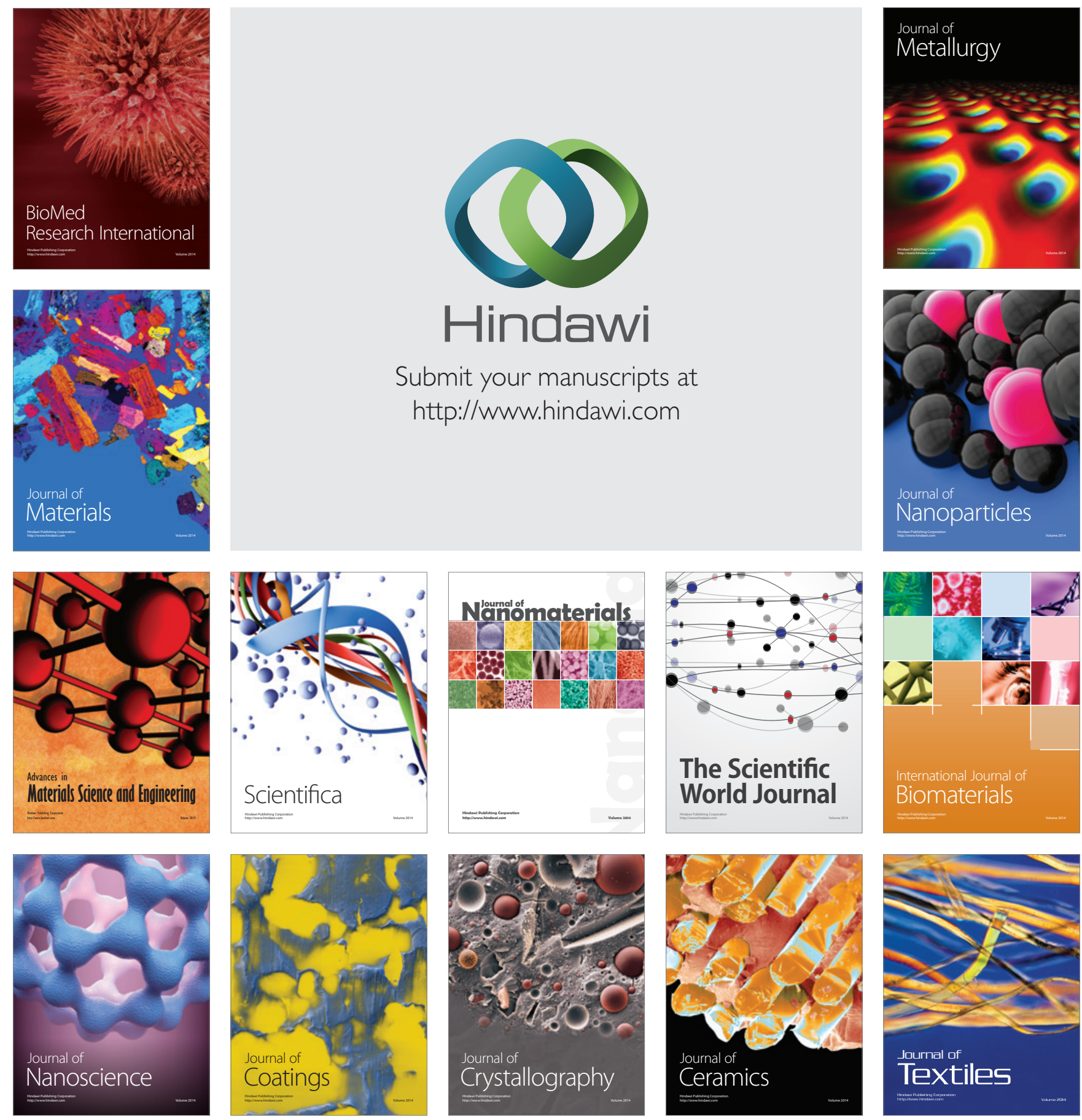